\title{
Clinical and laboratory features and response to treatment in patients presenting with vitamin $B_{12}$ deficiency-related neurological syndromes
}

\author{
S. Aaron, S. Kumar, J. Vijayan, J. Jacob, M. Alexander, C. Gnanamuthu \\ Neurology Unit, Department of Neurological Sciences, Christian Medical College, Vellore, Tamil Nadu, India
}

\begin{abstract}
Aims and objectives: To study the clinical and laboratory features of patients admitted with vitamin $B_{12}$ deficiency-related $\left(B_{12}\right.$ def) neurological syndromes. Settings and Design: A hospital-based retrospective and prospective study conducted at a referral teaching hospital. Materials and Methods: Consecutive patients admitted with vitamin $B_{12}$ deficiency-related neurological disorders during a three-year period from June 2000 to May 2003 were included. Data regarding clinical and laboratory features were obtained. Follow-up was done at least six months following treatment with parenteral vitamin $B_{12}$. Chi-square test was used for statistical analysis. Results: A total of 63 patients (52 males) with a mean age of 46.2 years were studied. The mean duration of symptoms at presentation was 10.3 months. Myeloneuropathy $(54 \%)$ was the commonest neurological manifestation, followed by myeloneuropathy with cognitive dysfunction (34\%), and peripheral neuropathy $(9 \%)$. Neuropsychiatric manifestations and dementia were observed in $38 \%$ and $19 \%$ of patients respectively. All the patients had megaloblastic changes in the bone marrow smear. Eleven (17.5\%) patients had both hemoglobin and the mean corpuscular volume (MCV) within the normal range. Follow-up after at least six months of therapy with parenteral B12 showed improvement in 54\% patients. Conclusions: $A$ high index of suspicion of $B_{12}$ def is required in patients presenting with myelopathy, cognitive decline, or neuropathy. A normal hemoglobin or MCV does not exclude $B_{12}$ def; therefore, other tests such as bone marrow smear and serum vitamin $B_{12}$ assay are essential, as the condition is often reversible with treatment.
\end{abstract}

Key Words: Bone marrow smear, Vitamin B12 deficiency, Myeloneuropathy

\section{Introduction}

Vitamin $\mathrm{B}_{12}$ deficiency $\left(\mathrm{B}_{12}\right.$ def) leads to myriad neurologi- cal manifestations and affects all age groups. ${ }^{[1]}$ Early recognition of this condition is essential, as it is reversible and preventable. ${ }^{[2]}$ However, a significant diagnostic delay still occurs in many cases. Though there is much debate regarding the appropriate method of diagnosing vitamin $\mathrm{B}_{12}$ def, the diagnosis is often made on the basis of a low serum vitamin $\mathrm{B}_{12}$ level or megaloblastic bone marrow or both. ${ }^{[2,3]}$ There are conflicting data regarding the recovery of patients with $\mathrm{B}_{12}$ def presenting with neurological involvement. ${ }^{[4]}$ This study was aimed at determining the common clinical presentations and outcome with treatment of patients admitted with $\mathrm{B}_{12}$ def.

\section{Materials and Methods}

This study was conducted in the Department of Neurological Sciences of a tertiary care teaching hospital. Retrospective and prospective descriptive study was carried out over a period of three years from June 2000 to May 2003 on consecutive patients admitted with a diagnosis of $\mathrm{B}_{12}$ def-related neurological syndromes. Data were collected regarding the demographic, clinical and laboratory features. All the patients underwent detailed general and neurological examination to identify the clinical syndromes. Patients were screened for dementia by mini-mental status examination (MMSE). The diagnosis of dementia was established on the basis of DSM-IV criteria. In addition, patients suspected to have cognitive impairment underwent a detailed assessment of various cognitive domains as per the neuropsychological proforma formulated at our department. ${ }^{[5]}$ Other primary degenerative dementias were excluded as per established diagnostic criteria. The diagnosis of $\mathrm{B}_{12}$ def was made on the basis of low serum vitamin $\mathrm{B}_{12}$ levels $(<200 \mathrm{pg} / \mathrm{ml})$ assessed by radioimmunoassay or presence of megaloblastic changes in the bone marrow smear or both. ${ }^{[2,3]}$ Appropriate electrophysiological and neuroimaging studies were also performed.

Patients were treated with intramuscular $\mathrm{B}_{12}$ injections (cyanocobalamin form, Neurobion forte, Merck India) at a dose of 1000 micrograms per day for seven days and then once a week for one month and thereafter once a month long-term. They were followed up for a minimum of six months and the response to therapy was 
noted. Assessment included a detailed general and neurological examination. In addition, laboratory parameters, neuroimaging and electrophysiological studies were repeated as per the clinical presentation. Functional status was assessed as per Barthel's index. Pearson's Chi-square test was used for statistical analysis.

\section{Results}

\section{Demographic Data}

A total of 63 patients (52 males) with a mean age of 46.2 years were studied. Majority $(31,49 \%)$ belonged to the age group of 31 to 50 years.

The mean duration of symptoms at presentation was 10.3 months (range 2-28 months).

The majority of patients belonged to a higher socioeconomic background (based on occupation, family assets and monthly income). Professionals and skilled workers constituted $70 \%$ of the patient population.

\section{Risk factors}

Predisposing factors were found in only 14 (22\%) patients. History of alcohol abuse (10 patients) and chronic diarrhea (6 patients) were the common underlying factors noted. Malabsorption work-up was positive in four patients. The majority were vegetarians $(60 \%)$. One patient had an ileal resection.

\section{Clinical features}

The common systemic features were fatigue (34\%), glossitis $(31 \%)$, weight loss $(27 \%)$ and anorexia (22\%) (Table 1). It was noted that those with fatigue had a lower hemoglobin level (mean $10.6 \mathrm{gm} \%$, SD \pm 2.58 ) when compared to those without fatigue (mean $12.4 \mathrm{gm} \%$, SD \pm 2.20 ), which was statistically significant $(P=0.005)$.

Skin and mucosal changes were seen in 26 patients, $47 \%$ of them (19\% of total) had cutaneous hyperpigmentation. Other findings included hair changes (9\%), angular stomatitis $(8 \%)$ and vitiligo $(3 \%)$. Even though hyperpigmentation was an important general examination finding, it did not correlate

\begin{tabular}{|c|c|c|}
\hline \multicolumn{3}{|c|}{$\begin{array}{l}\text { Table 1: Clinical presentation of patients with vitamin } B_{12} \\
\text { deficiency in our study }\end{array}$} \\
\hline & $\begin{array}{c}\text { Number } \\
(n=63)\end{array}$ & Percentage \\
\hline Myeloneuropathy & 34 & 54 \\
\hline Myeloneuropathy with cognitive impairment & 21 & 34 \\
\hline Cognitive impairment alone & 2 & 3 \\
\hline Peripheral neuropathy alone & 6 & 9 \\
\hline Neuropsychiatric manifestations & 24 & 38 \\
\hline Dementia & 12 & 19 \\
\hline Depression & 7 & 11 \\
\hline Psychosis & 5 & 8 \\
\hline Skin and mucosal changes & 26 & 41 \\
\hline Skin hyperpigmentation & 12 & 19 \\
\hline Hair changes & 6 & 9 \\
\hline Angular stomatitis & 5 & 8 \\
\hline Vitiligo & 2 & 3 \\
\hline
\end{tabular}

with duration of symptoms, severity of the megaloblastic change in the bone marrow smear, or the mean corpuscular volume (MCV).

Myeloneuropathy was the commonest clinical finding in 34 $(54 \%)$ patients. A combination of myeloneuropathy with cognitive impairment was seen in $21(34 \%)$ patients. Six $(9 \%)$ patients had peripheral neuropathy alone.

Cognitive dysfunction as the only manifestation of $\mathrm{B}_{12}$ def was noted in two $(3 \%)$ patients. Neuropsychiatric manifestations were noted in $24(38 \%)$ patients and included dementia in $19 \%$, depressive features in $11 \%$ and psychosis in $4 \%$. The mean MMSE score in patients with dementia was $15.5 \pm 7.5$. All patients with dementia had memory impairment. Language impairment was the next most common finding, affecting $77 \%$ of patients. Attention and concentration were unaffected in all the patients. Disorientation to space and person was seen in $55 \%$ of patients. Poor judgment and visuospatial disorientation were seen in $43 \%$ and $37 \%$ of patients respectively. All patients had features of temporal lobe dysfunction, whereas only $75 \%$ of patients with dementia had features of frontal and/or parietal lobe dysfunction. The occurrence of dementia in patients with $\mathrm{B}_{12}$ def did not correlate with duration of symptoms, or the severity of megaloblastic changes in the bone marrow smear.

\section{Laboratory features}

All the patients had megaloblastic changes in the bone marrow smear. Mild changes were noted in $56 \%$ while moderate and severe changes were noted in $22 \%$ each (Table 2). Hypersegmented neutrophils in the peripheral blood were seen in only 10 patients $(16 \%)$. The mean hemoglobin was 11.3 Gm \%, MCV 103.1 Fl and the serum LDH 871 U/L respectively. Eleven patients (17.5\%) had both their hemoglobin and the $\mathrm{MCV}$ within the normal range $(\mathrm{Hb}>12 \mathrm{Gm} \%$ and the $\mathrm{MCV}<98 \mathrm{Fl})$. Clinical syndromes in them included

Table 2: Laboratory abnormalities in patients with vitamin $B_{12}$ deficiency in our study

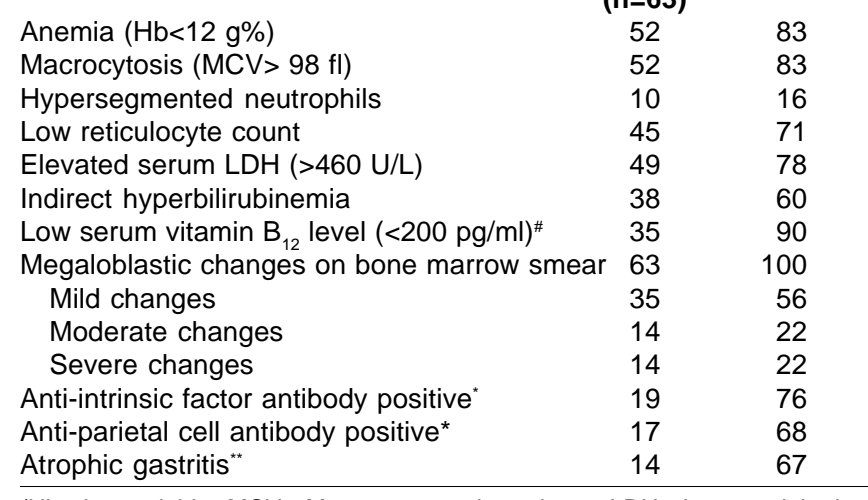

$(\mathrm{Hb}=$ hemoglobin; $\mathrm{MCV}=$ Mean corpuscular volume; $\mathrm{LDH}=$ Lactate dehydrogenase); \#: Serum $B_{12}$ level was tested in only 39 patients; *: Anti-intrinsic factor and anti-parietal cell antibodies were tested in 25 patients; ${ }^{\star *}$ : Upper gastrointestinal endoscopy and biopsy were done in 21 patients. 
myeloneuropathy in seven, peripheral neuropathy in three and dementia in one.

The serum B12 levels were low (normal 200-950 pg/ml) in 35 patients (tested in 39 patients). Serum folate level was within the normal range $(3-17 \mathrm{ng} / \mathrm{ml})$.

Serum homocysteine was elevated in 13 patients (tested in 14). Anti-intrinsic factor antibody was positive in $19(76 \%)$ and anti-parietal cell antibody was positive in 17 (68\%) out of 25 patients, in whom they were tested. Upper gastrointestinal endoscopy was performed in 21 patients and showed features of atrophic gastritis in 14 (66\%). Malabsorption work-up was positive in four patients.

\section{Neuroimaging and Electrophysiological Findings}

The neuroimaging findings are summarized in Table 3. Findings of the nerve conduction studies are summarized in Table 4. Visual evoked potentials showed features of optic nerve dysfunction in 16 (out of 29) patients. Brainstem auditory evoked responses were abnormal in 3 (out of 21). Somatosensory evoked responses (tested in 23 patients) showed features of cervical cord dysfunction in 14 and dorsal cord dysfunction in 17 patients.

\section{Follow-up}

Follow-up after a mean duration of 13.4 months (range 634 months) after starting therapy with parenteral $\mathrm{B}_{12}$ showed improvement in $33(54 \%)$ patients. There was no significant change in nine (14\%) and $21(32 \%)$ were lost to follow-up.

Thus, in effect, $79 \%(33 / 42)$ of the patients who could be evaluated after six months had shown improvement. Cognitive functions showed a significant improvement. Mean MMSE scores improved by 2.4 to a level of $17.9 \pm 6.4(P<0.01 ; 95 \%$ Confidence Interval (CI): -3.9 to -1.6$)$. Functional status had markedly improved in them. The 'activities of daily living score' in the Barthel index significantly increased from a pretreatment level of $62.6 \pm 33.2$ to $75.7 \pm 18.4(P<0.01 ; 95 \%$ CI:

\begin{tabular}{lccc}
\hline \multicolumn{4}{c}{ Table 3: Summary of the neuroimaging findings in patients } \\
included in this study \\
\hline Findings & $\begin{array}{c}\text { CT Brain } \\
(\mathbf{n}=\mathbf{9})^{*}\end{array}$ & $\begin{array}{c}\text { MRI Brain } \\
(\mathbf{n}=\mathbf{1 4})^{*}\end{array}$ & $\begin{array}{c}\text { MRI Spine } \\
(\mathbf{n}=\mathbf{3 9})\end{array}$ \\
Normal & 6 & 5 & 17 \\
Atrophy & 3 & 6 & 3 \\
White matter lesions & 1 & 3 & 19 \\
Lacunar infarcts & 1 & 2 & 0 \\
\hline
\end{tabular}

(CT= Computerized tomography; MRI= Magnetic Resonance Imaging); *Some patients had more than one imaging abnormality.

\section{Table 4: Summary of the nerve conduction studies of patients} included in our study*

\section{Normal}

Axonal sensori-motor neuropathy

Demyelinating sensori-motor neuropathy

Mixed (axonal and demyelinating) sensori-motor neuropathy

*Nerve conduction studies were performed on 49 patients.
-24.8 to -8.3$)$ after the treatment. The response to the treatment was not affected by the severity of the megaloblastic changes in the bone marrow or the duration of the neurological symptoms.

\section{Discussion}

Even though the human body has enough vitamin $B_{12}$ stores to last for up to five years, its deficiency is not uncommon. The main systems affected due to $\mathrm{B}_{12}$ def are the hematological, skin and mucous membranes, and the nervous system. ${ }^{[6]} \mathrm{Neu-}$ rological features are attributable to pathology in the peripheral nerves, optic nerves, posterior and lateral columns of the spinal cord and brain. $B_{12}$ def is a classic neurological 'systemspecific degeneration' in which particular sets of neurons are affected because of their selective vulnerability. An increased prevalence of $\mathrm{B}_{12}$ def has been reported in patients infected with the human immunodeficiency virus (HIV). ${ }^{[7]}$ However, none of our patients had HIV infection. $\mathrm{B}_{12}$ def causes a wide spectrum of neurological manifestations ranging from neural tube defects ${ }^{[8]}$ to changes in cognition and behavior. ${ }^{[9]}$ Many unusual manifestations have been reported in infants ${ }^{[10]}$ and adults including movement disorders ${ }^{[11]}$ and seizures. ${ }^{[12]}$

The diagnosis in our cases was based on either a megaloblastic bone marrow or low serum vitamin $\mathrm{B}_{12}$ level or both. A megaloblastic bone marrow was taken as a definitive evidence of $B_{12}$ def because: (i) some patients may have received $B_{12}$ supplements prior to their referral to us which can influence the serum $\mathrm{B}_{12}$ levels; (ii) in a vitamin $\mathrm{B}_{12}$-deficient state, the levels first fall in the neuronal tissues and only much later is it reflected in the serum vitamin $\mathrm{B}_{12}$ levels. Therefore, a diagnosis of $\mathrm{B}_{12}$ def might be missed if we solely rely on the serum levels; (iii) the presence of megaloblasts in the bone marrow is virtually diagnostic of vitamin $\mathrm{B}_{12}$ or folate deficiency. However, a serum vitamin $B_{12}$ assay is mandatory in all cases of suspected $\mathrm{B}_{12}$ def.

A referral bias probably may be the reason behind the higher proportion of inclusion of males $(83 \%)$, and those from a higher socioeconomic background $(70 \%)$ in this study. However, it could also be due to the fact that the etiology of $\mathrm{B}_{12}$ deficiency seems to be immune-mediated in a significant number of patients and an autoimmune process could affect more affluent people as well. Vegetarians constituted $60 \%$ of the study population. Earlier Indian studies have also highlighted this. ${ }^{[13,14]}$ This finding confirms that vegetarians are at a higher risk of developing $\mathrm{B}_{12}$ def, which is probably corroborated by the finding that vegetarians had a statistically more severe form of megaloblastic changes in the bone marrow as compared to the non-vegetarians.

Even though the classical syndrome of $\mathrm{B}_{12}$ def is termed 'subacute' combined degeneration of the cord, a wide variation is noted in the duration of symptoms at the time of presentation (2 months-28 months). The deficiency can also occur acutely 
following exposure to nitrous oxide. ${ }^{[15,16]}$

Fatigue was the commonest symptom in this series. $\mathrm{B}_{12}$ def has been implicated in conditions where fatigue is a prominent symptom such as chronic fatigue syndrome. ${ }^{[17]}$ In this study, fatigue was associated with a low hemoglobin level.

Hyperpigmentation over the extremities, especially over the dorsum of the hands and feet with accentuation over the terminal phalanges and inter-phalangeal joints, associated with pigmentation of the oral mucosa is characteristic of $\mathrm{B}_{12}$ def. These findings resolve with therapy. ${ }^{[18]}$

Myeloneuropathy was the commonest neurological manifestation in this study. In another similar study from the West, diminished vibratory sensation and proprioception in the lower extremities were the most common objective neurological findings. ${ }^{[1]}$ This difference may be due to the delayed presentation of patients to our center in this study. The median duration of symptoms in our patients was 10.3 months as compared to 4 months in the Western study.

The association between neuropsychiatric disorders and $\mathrm{B}_{12}$ def is well known. Dementia in our study did not correlate with either the duration of symptoms or the severity of the megaloblastic changes in the bone marrow smear. These results are similar to the findings reported by Lindenbaum et al, ${ }^{[19]}$ where it was shown that the neuropsychiatric disorders due to cobalamin deficiency occurred commonly in the absence of anemia or macrocytosis. A high prevalence of low serum vitamin $\mathrm{B}_{12}$ levels, and other indicators of vitamin $\mathrm{B}_{12}$ deficiency have been reported among people with Alzheimer's disease and older people. However, evidence of any efficacy of vitamin $\mathrm{B}_{12}$ therapy in improving the cognitive function of people with dementia and low serum $\mathrm{B}_{12}$ levels is insufficient. ${ }^{[20]}$

It was interesting to note that 11 patients $(17.5 \%)$ had both their hemoglobin and the MCV within the normal range. Other studies both from India and the West have reported similar findings. ${ }^{[19-22]}$ Wadia et $\mathrm{al}^{[13]}$ had shown that hematological parameters alone are likely to miss nearly $50-70 \%$ of the cases of $\mathrm{B}_{12}$ def. They used a cut-off of $\mathrm{Hb}<11 \mathrm{gm} \%$ and $\mathrm{MCV}<$ $95 \mathrm{fl}$ in their study, as compared to a slightly higher cut-off taken in this study $(\mathrm{Hb}<12 \mathrm{gm} \%$ and the $\mathrm{MCV}<98 \mathrm{fl})$.

After a follow-up of six months or more, 54\% showed improvement. This could be higher in reality, as we assumed that all the cases that were lost to follow-up might not have improved. The response to treatment was not affected by the severity of the megaloblastic changes in the bone marrow or the duration of symptoms. This finding was contrary to the findings of Lindenbaum et al, ${ }^{[19]}$ who showed that the degree of improvement over the baseline neurological status after treatment was inversely related to the duration of symptoms and hematocrit. Functional status also improved as assessed by the Barthel index. However, it should be noted that Barthel's index may not be appropriate for assessing the outcome of varied neurological syndromes including peripheral neuropa- thy. This could be a limitation to generalizing the results of this study.

A high index of suspicion of $\mathrm{B}_{12}$ def is needed in patients presenting with myelopathy, cognitive decline, or neuropathy, especially among the pure vegetarians and the elderly. Skin changes might be an important clue towards the diagnosis. The duration of symptoms is highly variable. A normal hemoglobin and MCV, and even a normal serum $\mathrm{B}_{12}$ level does not rule out $\mathrm{B}_{12}$ def; hence other tests like bone marrow smear might be useful in diagnosing this condition, as it is reversible in a majority.

\section{References}

1. Healton EB, Savage DG, Brust JC, Garrett T.J, Lindenbaum J. Neurologic aspects of cobalamin deficiency. Baltimore: Medicine; 1991;70:229-45.

2. Misra UK, Kalita J, Das A. Vitamin B12 deficiency neurological syndromes: A clinical, MRI and electrodiagnostic study. Electromyogr Clin Neurophysiol 2003;43:57-64.

3. Pandey S, Kalita J, Misra UK. A sequential study of visual evoked potential in patients with vitamin B12 deficiency neurological syndrome. Clin Neurophysiol 2004;115:914-8.

4. Moretti R, Torre P, Antonello RM, Cattaruzza T, Cazzato G, Bava A. Vitamin B12 and folate depletion in cognition: A review. Neurol India 2004;52:310-8.

5. Kumar S, Hannah V, Alexander M, Gnanamuthu C. Rapidly progressive dementia as a presenting feature of acute disseminated encephalomyelitis. Ann Ind Acad Neurol 2003;6:167-70.

6. Baker S.J. Human Vitamin B12 Deficiency: World Review of Nutrition and Dieteties. 1967;8:62-126.

7. Kieburtz KD, Giang DW, Schiffer RB, Vakil N. Abnormal vitamin B12 metabolism in human immunodeficiency virus infection. Association with neurological dysfunction. Arch Neurol 1991;48:312-4.

8. Kirke PN, Molloy AM, Daly LE, Burke H, Weir DG, Scott JM. Maternal plasma folate and vitamin B12 are independent risk factors for neural tube defects. Q J Med 1993;86:703-8

9. Shorvon SD, Carney MW, Chanarin I, Reynolds EH. The neuropsychiatry of megaloblastic anaemia. Br Med J 1980;281:1036-8.

10. Jadhav M, Webb JK, Vaishnava S, Baker S.J. Vitamin B12 deficiency in Indian infants. A clinical syndrome. Lancet 1962;2:903-7.

11. Kumar S. Vitamin B12 deficiency presenting with an acute reversible extrapyramidal syndrome. Neurol India 2004;52:507-9.

12. Kumar S. Recurrent seizures: An unusual manifestation of vitamin B12 deficiency. Neurol India 2004;52:122-3.

13. Dastur DK. Quadros EV, Wadia NH, Desai MM, Bharucha EP. Effect of vegetarianism and smoking on vitamin B12, thiocyanate, and folate levels in the blood of normal subjects. Br Med J 1972;3:260-3.

14. Wadia RS. Kharche M. Udar M, Kulkarni S, Kothari S, Karve S. Vitamin $\mathrm{B}_{10}$ and folate deficiency in a hospital population. Ann Ind Acad Neurol 2001;4:19-25.

15. Amess JA, Burman JF, Rees GM, Nancekievill DG, Mollin DL. Megaloblastic haemopoiesis in patients receiving nitrous oxide. Lancet 1978;2:339-42.

16. Amos RJ, Amess JA, Hinds CJ, Mollin DL. Incidence and pathogenesis of acute megaloblastic bone-marrow change in patients receiving intensive care. Lancet $1982 ; 2: 835-8$.

17. Lawhorne L, Ringdahl D. Cyanocobalamin injections for patients without documented deficiency. Reasons for administration and patient responses to proposed discontinuation. JAMA 1989;261:1920-3.

18. Baker SJ, Ignatius M, Johnson S, Vaish SK. Hyperpigmentation of skin. A sign of vitamin-B12 deficiency. Br Med J 1963;5347:1713-5.

19. Lindenbaum J, Healton EB, Savage DG, Brust JC, Garrett T.J, Podell ER, et al. Neuropsychiatric disorders caused by cobalamin deficiency in the absence of anemia or macrocytosis. N Engl J Med 1988;318:1720-8.

20. Malouf R, Areosa SA. Vitamin B12 for cognition. Cochrane Database Syst Rev 2003;CD004326.

21. Strachan RW, Henderson JG. Psychiatric syndromes due to avitaminosis B12 with normal blood and marrow. Q J Med 1965;34:303-17.

22. Carmel R. Pernicious anemia. The expected findings of very low serum cobalamin levels, anemia, and macrocytosis are often lacking. Arch Intern Med $1988 ; 148: 1712-4$.

Accepted on 09.11.2004. 


\section{Invited Comments}

It is today well known that vitamin $B_{12}$ deficiency can be associated with neuropsychiatric symptoms. Several studies have previously demonstrated that vitamin $B_{12}$ deficiency is more common in patients with dementia symptoms than in the cognitively non-impaired. This topic is touched upon in the article "Clinical and laboratory features and response to treatment in patients presenting with vitamin $\mathrm{B}_{12}$ deficiencyrelated neurological syndromes" in the current issue of Neurology India. Vitamin $\mathrm{B}_{12}$ deficiency increases with age and is present in $5-40 \%$ of the elderly population. However, the mechanism of neurological damage induced by a quantitative or functional vitamin $\mathrm{B}_{12}$ deficiency is still unclear.

Vitamin $B_{12}$ exerts its physiological effect on two major enzymatic pathways: the conversion of homocysteine to methionine and the conversion of methylmalonyl coenzyme A to succinyl coenzyme A. Disruption of either of these pathways due to vitamin $B_{12}$ deficiency results in an elevation of both serum homocysteine and methylmalonic acid. Homocysteine levels are also elevated in the case of folate deficiency. Serum homocysteine is proposed to be more sensitive for functional intracellular vitamin $\mathrm{B}_{12}$ deficiency than analysis of vitamin $\mathrm{B}_{12}$ in serum. ${ }^{1}$ Hence, homocysteine, vitamin $\mathrm{B}_{12}$, and folate are closely linked together in the so-called one-carbon cycle. The proposed mechanism relates to the methylation reactions involving homocysteine metabolism in the nervous system. ${ }^{2}$ Smith $^{3}$ has suggested that the brain suffers from a double whammy from hyperhomocysteinaemia: cerebrovascular damage that triggers or potentiates the effect of Alzheimer pathology combined with a direct neuro- toxic effect of homocysteine.

There is an ongoing debate about the contribution of vita$\min \mathrm{B}_{12}$ deficiency to the dementia syndrome. The current article adds to those finding an effect on cognitive function as a result of vitamin $B_{12}$ treatment in patients with cognitive impairment and co-occurring vitamin $\mathrm{B}_{12}$ deficiency. One of the most intriguing findings in the study is the low mean age of the investigated subjects. It must be considered unexpected to find such a high percentage of cases with cognitive symptoms in ages below 65 years. It would be very interesting to see added neuropsychological data on the dementia cases, to elucidate exactly in what cognitive domains there was an improvement. It is possible that such a study might contribute to a better understanding of role of vitamin $B_{12}$ deficiency in dementia evolution.

\section{Katarina Nagga}

Department of Geriatric Medicine, University Hospital, SE-581 85 Linkoping, Sweden. E-mail: katarina.nagga@lio.se

\section{References}

1. Joosten E, Van den Berg A, Riezler R, Naurath HJ, Lindenbaum J, Stabler $\mathrm{SP}$, et al. Metabolic evidence that deficiencies of vitamin $\mathrm{B}_{12}$ (cobalamin), folate, and vitamin $\mathrm{B}_{6}$ occur commonly in elderly people. Am $\mathrm{J}$ Clin Nutr 1993;58:468-76.

2. Santosh-Kumar C, Hassell K, Deutsch J, Kolhouse J. Are neuropsychiatric manifestations of folate, cobalamin, and pyridoxine deficiency mediated through imbalances in excitatory sulfur amino acids? Med Hypotheses $1994 ; 43: 239-44$

3. Smith AD. Homocysteine, B vitamins, and cognitive deficit in the elderly. Am J Clin Nutr 2002;75:785-6. 